\title{
Role of HDAC6 inhibition in sepsis-induced acute respiratory distress syndrome (Review)
}

\author{
QINGHUA ZHANG, YAN WANG, DANHUA QU, JINYAN YU and JUNLING YANG \\ Department of Respiratory and Critical Care Medicine, The Second Hospital \\ of Jilin University, Changchun, Jilin 130041, P.R. China
}

Received August 21, 2020; Accepted February 1, 2021

DOI: $10.3892 /$ etm.2021.9866

\begin{abstract}
Acute respiratory distress syndrome (ARDS) induced by sepsis contributes remarkably to the high mortality rate observed in intensive care units, largely due to a lack of effective drug therapies. Histone deacetylase 6 (HDAC6) is a class-IIb deacetylase that modulates non-nuclear protein functions via deacetylation and ubiquitination. Importantly, HDAC6 has been shown to exert anti-cancer, anti-neurodegeneration, and immunological effects, and several HDAC6 inhibitors have now entered clinical trials. It has also been recently shown to modulate inflammation, and HDAC6 inhibition has been demonstrated to markedly suppress experimental sepsis. The present review summarizes the role of HDAC6 in sepsis-induced inflammation and endothelial barrier dysfunction in recent years. It is proposed that HDAC6 inhibition predominantly ameliorates sepsis-induced ARDS by directly attenuating inflammation, which modulates the innate and adaptive immunity, transcription of pro-inflammatory genes, and protects endothelial barrier function. HDAC6 inhibi-
\end{abstract}

Correspondence to: Dr Jinyan $\mathrm{Yu}$ and Dr Junling Yang, Department of Respiratory and Critical Care Medicine, The Second Hospital of Jilin University, 218 Ziqiang Street, Changchun, Jilin 130041, P.R. China

E-mail: yujinyan1988@163.com

E-mail: junling@jlu.edu.cn

Abbreviations: ARDS, acute respiratory distress syndrome; PEEP, positive and end-expiratory pressure; HDAC6, histone 6; HAT, histone acetyltransferase; HSP90, heat shock protein 90; LPS, lipopolysaccharide; Edn, endothelin; IL, interleukin; CLP, cecal ligation and puncture; TNF- $\alpha$, tumor necrosis factor; MPO, myeloperoxidase; CCL-2, C-C motif ligand 2; CXC, $\mathrm{C}-\mathrm{X}-\mathrm{C}$ motif; MAPK, mitogen-activated protein kinase; ERK, extracellular-signal-regulated kinase; JNK, c-Jun N-terminal kinase; $\mathrm{NF}-\kappa \mathrm{B}$, nuclear factor- $\kappa \mathrm{B}$; AP, activator protein; HIV, human immunodeficiency virus; Tat, transactivator of transcription; IRF-3, interferon regulatory factor 3; IFN, interferon; MLC, myosin long chain; EBD, endothelial barrier dysfunction; AJ, adherens junction; TJ, tight junction; VE, vascular endothelial; TubA, tubastatin A

Key words: HDAC6, sepsis, ARDS, inflammation, EBD tion protects against sepsis-induced ARDS, thereby making HDAC6 a promising therapeutic target. However, HDAC inhibition may be associated with adverse effects on the embryo sac and oocyte, necessitating further studies.

\section{Contents}

1. Introduction

2. Sepsis-induced ARDS and HDAC6

3. HDAC6 inhibition protects against inflammation

4. HDAC6 inhibition restores endothelial barrier hyper-permeability

5. A promising and highly selective HDAC6 inhibitor, TubA

6. Conclusion

\section{Introduction}

Acute respiratory distress syndrome (ARDS) was first described in 1967 by Ashbaugh et al (1) in a patient suffering from sudden onset of dyspnea that was resistant to standard oxygen therapy, accompanied by a loss of lung compliance and diffuse alveolar infiltrates. The original definition of ARDS has since been updated, and the disease is now commonly defined according to the 'Berlin' definition proposed by the European Society of Intensive Care Medicine ARDS Definition Task Force in 2012 (2). ARDS is considered a significant health and economic burden. The incidence of ARDS in the intensive care units (ICUs) of 50 countries was $10.4 \%$. Furthermore, the incidence per ICU bed for four weeks in Europe was 0.48; North America, 0.46; South America, 0.31; Asia, 0.27; Africa, 0.32; and Oceania, 0.57 cases, accounting for $23.4 \%$ of all patients who need mechanical ventilation. Despite advances in supportive treatment, the mortality of ARDS is still high. As the severity of ARDS increases, $40 \%$ of patients with ARDS died in hospitals (3). In China, the prevalence of ARDS among patients in ICUs can be as high as $4.5 \%$, with a mortality rate of $52 \%(4,5)$. Moreover, the long-term prognosis for ARDS survivors is poor; in fact, a previous study showed that at 5 years, up to $28 \%$ of survivors exhibited a decreased capacity for self-care, as well as various physiological and psychological sequelae, thereby requiring continuous treatment (4). Clinical ARDS treatment 
mainly comprises mechanical ventilation in a prone position and/or extracorporeal membrane oxygenation (6). Ventilator management varies with the severity of ARDS:35.1\% of patients generally have tidal volumes above $8 \mathrm{ml} / \mathrm{kg}$ predicted body weight, whereas $82.6 \%$ received positive and end-expiratory pressure (PEEP) of $<12 \mathrm{~cm} \mathrm{H}_{2} \mathrm{O}$. However, from mild to moderate to severe cases, the decrease in tidal volume and the increase in PEEP could be beneficial, and the data were statistically significant. A recent study also showed that, compared with a low PEEP strategy, higher PEEP did not significantly decrease baro trauma, new organ failure, or ventilator-free days (medium-level evidence) (7). Compared with control ventilation, maximal lung recruitment did not reduce the number of days without ventilation or mortality, which was associated with the increase in cardiovascular adverse events (8). Venous extracorporeal membrane oxygenation can be considered as a strategy for patients with ARDS at the early stages to receive lung protective ventilation. However, effective drug treatments are not available for ARDS, and ARDS-associated mortality and disability have not significantly decreased in recent years. Steroids may be the most widely studied and discussed drugs for ARDS treatment, with the results still being considered controversial. Previous studies $(9,10)$ have confirmed that patients with ARDS taking methylprednisolone did not result in decreased mortality, and prolonged steroid use was associated with an increased mortality compared with that in the placebo group. However, other studies provide contradicting evidence, demonstrating that patients receiving steroids (methylprednisolone or hydrocortisone) had a higher rate of spontaneous breathing on day 28 and lower in-hospital mortality, and early administration of dexamethasone as one can decrease the duration of mechanical ventilation and total mortality in patients with confirmed moderate to severe ARDS (11). Although it is possible that subgroups of patients with ARDS will benefit from steroids, it is unknown who these patients are. In addition, given the underlying pathological heterogeneity of patients with confirmed ARDS, those who respond may, in fact, have undiagnosed inflammatory lung disease, such as tissue pneumonia. The most common adverse effect of steroid use was hyperglycemia, while other potential side effects of therapeutic steroids include neuromuscular weakness, immunosuppression, severe infections, as well as sodium retention, which is associated with adverse outcomes (11). Thus, presently, steroids are not recommended for routine treatment of ARDS and further studies regarding their benefits in patients with ARDS are needed.

Of the various identified infectious and non-infectious factors, severe sepsis remains a primary cause of ARDS (12). Acute lung injury (ALI) is commonly used in experiments to study ARDS.

Histone acetyltransferases (HAT) and deacetylases (HDACs) are widely expressed in the airways, and they modulate histone acetylation and deacetylation, respectively (13). HDAC6 is a class-IIb HDAC that, uniquely, contains two independent catalytic domains (14-18). While both catalytic domains must remain intact to facilitate HDAC6-driven deacetylation, each selectively recognizes and interacts with specific HDAC6 substrates (17), including $\alpha$-tubulin, heat shock protein 90 (HSP90), and cortactin (19-22).
Notably, HDAC6 deregulation has been shown to mediate disease processes including carcinogenesis, neurodegeneration and autoimmunity $(14,15,23)$. Accordingly, several HDAC6 and pan-HDAC inhibitors have been approved for anti-neoplastic clinical trials (24-26). Recent studies have focused on the potential role of HDAC6 in inflammation, after Halili et al (27) first demonstrated that HDAC6 inhibition represses macrophage inflammatory responses to lipopolysaccharide (LPS). Several research groups have since revealed a role for HDAC6 in sepsis pathogenesis and have shown that its inhibition protects against both inflammation and sepsis-induced endothelial barrier dysfunction. These findings support further investigation of HDAC6 inhibition as a promising potential therapeutic strategy targeting HDAC6, thereby attenuating sepsis-induced ARDS.

\section{Sepsis-induced ARDS and HDAC6}

Sepsis is a systemic disease induced by an overwhelming infection, resulting in multiple organ system dysfunction, including ARDS. Inflammation and endothelial barrier dysfunction are important pathophysiological changes that promote the incidence and development of sepsis-induced ARDS.

ARDS/ALI is a group of clinically and biologically heterogeneous diseases. Various pathogenic factors induce increased pulmonary capillary permeability and cause pneumonia edema, leading to acute respiratory failure. The characteristic change associated with ARDS/ALI is refractory hypoxemia, which is difficult to be corrected through oxygen inhalation and whose common inducements are sepsis, aspiration pneumonia, shock and trauma, among others. Treatments include ventilator support, prone position ventilation, treatment of primary disease, and restriction of liquid intake. Despite these, ARDS/ALI still show high mortality with a lack of effective drug treatment (28). Furthermore, the pathogenesis of ALI is complex, involving a series of cytokines and inflammatory factors, which can cause vascular endothelial and alveolar epithelial cell damage, alveolar collapse, and pulmonary edema. At present, research on the mechanism of ALI induced by sepsis mainly includes inflammatory pathways, oxidative stress, apoptosis and endothelial barrier dysfunction. Inflammation is the protective native immune response of an organism to a pathogen or tissue injury, which can act as a double-edged sword; when overactivated or not activated properly, it can lead to some immune disorders, septic shock, rheumatoid arthritis and neurodegenerative diseases. Pathogenic factors induce inflammation by modulating signaling pathways such as the HMGB-RAGE-NF- $\kappa$ B and MAPK pathways to produce a large number of pro-inflammatory factors and mediators that can eventually cause sepsis. Oxidative stress is an important cause of multiple organ damage in sepsis (29), and plays an important role in ALI caused by various inducements. It can increase lung water content, damage the integrity of pulmonary endothelial barrier function, and lead to lung injury. At present, it is generally believed that decreasing oxidative stress can likewise lower lung injury (30-32). The important factors involved in oxidative stress include nitrous oxide, PGE2, iNOS, COX-2, SPD and other factors that cause damage to alveolar capillaries. Apoptosis is also an important 


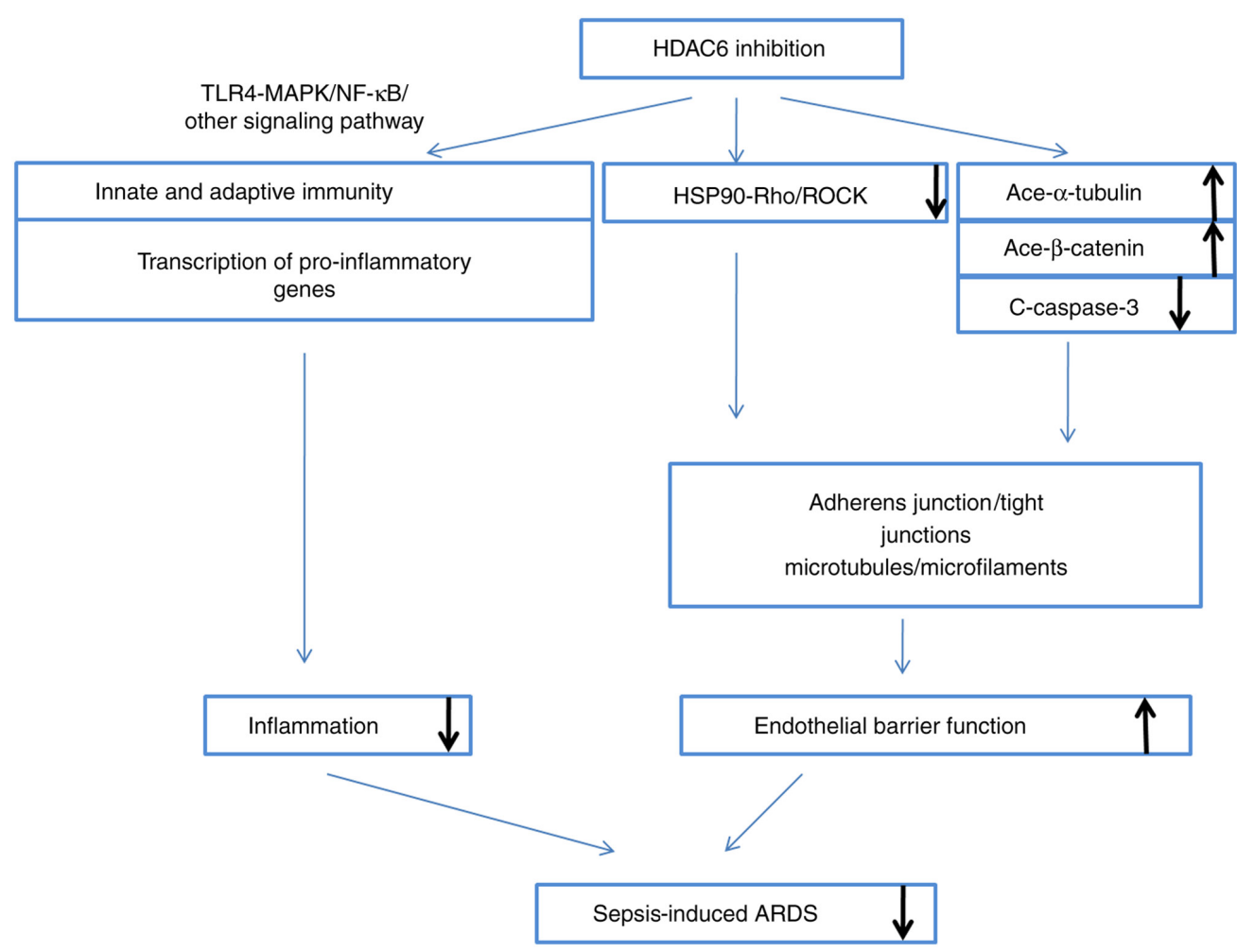

Figure 1. HDAC6 inhibition ameliorates sepsis-induced ARDS. The inhibition occurs mainly by two ways: i) Modulation of innate and adaptive immune cells, and the transcription of pro-inflammatory genes, via the TLR4-MAPK/NF-kB pathway, which directly attenuates inflammation; and ii) protection of endothelial barrier function via HSP90, $\alpha$-tubulin, $\beta$-catenin and caspase-3, thereby enhancing adherents and strengthening the cytoskeleton. HDAC6, histone

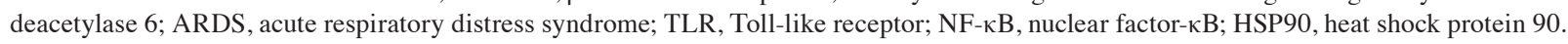

factor in the progression of sepsis-induced ARDS/ALI. On one hand, it directly destroys the integrity of the endothelial barrier through endothelial cell apoptosis, and on the other, it has been reported that the activity of apoptosis-associated proteins such as caspase- 3 can lead to the rearrangement of cell junction proteins (such as VE-cadherin and claudin-5). Lastly, in recent years, research on the function of endothelial cell barrier in ALI has gradually increased. At present, one of the main signaling pathways found to be involved in endothelial cell barrier function is the S1P-SIP1 signaling pathway; with the activation of Gi protein-combined SIP1 receptor and downstream small GTPase Rac molecule, S1P induces cortactin transfer and peripheral myosin long chain (MLC) phosphorylation, which can strengthen the rearrangement of cytoskeleton structure and form a cortical actin ring, and strengthen the adhesion connection, tight connection, and local adhesion complex on the cell surface, thereby decreasing pulmonary edema induced by LPS in ALI (33). In addition, the following are some pathways and systems that are also involved: Ang-2-Tie-2-MLC, renin-angiotensin system (34), Rho-ROCK-LIMK1-MLC, eNOS-Cav1-MLC2 signaling (35), PAR1 moesin (36), HDAC6-Hsp90 and $\alpha$-tubulin- $\beta$-catenin (37-39).

HDAC6 is extensively expressed in normal airways, where it promotes microtubule destabilization and endothelial barrier hyper-permeability (40). Accordingly, HDAC6 inhibition has been shown to improve survival in murine sepsis models, either by blocking endothelial barrier hyper-permeability or by attenuating airway inflammation (41-46), as will be further discussed (Fig. 1).

\section{HDAC6 inhibition protects against inflammation}

Halili et al (27) first demonstrated that the HDAC6 inhibitor 17a effectively inhibits LPS-induced pro-inflammatory endothelin (Edn)-1 and interleukin (IL)-12p40 RNA expression in macrophages. Various studies have since shown that HDAC6 directly mediates inflammation and both the innate (i.e., pathogen sensing and destruction) and adaptive immunity, as well as the modulation of the transcription of pro-inflammatory genes $(47,48)$.

Role of HDAC6 in innate and adaptive immunity cells. Inflammation is a protective innate immune response to invading pathogens and helps to prevent tissue damage. It acts like a 'double-edged sword' and, upon overactivation or improper activation, causes serious immune disorders, systemic inflammatory response syndrome, compensatory anti-inflammatory response syndrome, innate immune and adaptive immune disorders, and subsequently leads to multiple organ dysfunction such as ARDS. The process of inflammation begins with the influx of neutrophils, followed by the recruitment of monocytes, which then differentiate into inflammatory macrophages or dendritic cells. These cells are the key effector cells in the inflammatory site, recognizing and phagocytizing pathogens and necrotic cells to help eliminate infection. In addition, they activate the adaptive immune response through communication and coordination with other immune cells. Macrophages are the main effector cells in the inflammatory response and play a key role in initiation, regression, tissue repair, and regeneration of the inflammatory response (49). 
Monocytes and macrophages, as the most efficient pathogen scavengers and the main source of inflammatory cytokines, are the key effector cells that modulate the innate immune response of the body. However, the progressive dysfunction of monocytes and macrophages also leads to immune dysfunction during severe sepsis and septic shock. Thus, HDAC6 inhibition attenuates macrophage-induced inflammation by inhibiting the overproduction of reactive oxygen species (ROS) and modulating the expression of pro-inflammatory cytokines induced by LPS (50). A study has also shown that HDAC6 deficiency impairs macrophage recruitment to the inflammation site in a murine model of acute peritonitis, which may suppress the phagocytic capacity of macrophages, but at the same time serves as a promising therapeutic strategy for the treatment of macrophage-associated immune diseases (49); nonetheless, further research is still needed. The increased preoperative neutrophil lymphocyte ratio and high and middle ratio of neutrophils can have poor prognosis in patients with cancer. In addition, the proportion of neutrophils are found to be significantly higher, with the lymphocyte count significantly lower, in critical patients with severe sepsis or septic shock. According to the sequential organ failure assessment (SOFA) and acute physiological and chronic health assessment II (APACHE II) scores, the severity of the clinical course is associated with the difference in the percentage of neutrophils and lymphocytes in leukocytes. A selective HDAC6 inhibitor, tubastatin A (TubA) has been demonstrated to alter the composition of blood cells, restore the lymphocyte population, and decrease the granulocyte-to-lymphocyte ratio by improving the monocyte and granulocyte count in a murine model of cecal ligation and puncture (CLP), providing another explanation for the increase insurvival outcome in septic death model. Furthermore, selective HDAC6 inhibition is needed to restore innate immune cells in the bone marrow, lower stress responses, immune organ atrophy and apoptosis $(43,48,51,52)$.

HDAC6 modulates pro-inflammatory gene transcription. Cytokines, chemokines and adhesion molecules are important pro-inflammatory mediators, and HDAC6 overexpression increases the production of pro-inflammatory factors in macrophages. Conversely, HDAC6 inhibition suppresses their expression and circulation, such as tumor necrosis factor (TNF)- $\alpha$ and IL-6 in peritoneal fluids, decreases myeloperoxidase (MPO) production, and increases IL-10 production $(45,50,53)$. HDAC6 inhibition has also been reported to decrease the expression of adhesion molecules and chemokines, including C-C motif ligand 2 (CCL-2), C-X-C motif (CXC)L-8 and CXCL-10. Additionally, HDAC6 represses extracellular signal-regulated kinase (ERK), c-Jun N-terminal kinase (JNK), p38 and nuclear factor (NF)- $\kappa$ B. HDAC6 also represses activator protein (AP)-1 activation in astrocytes responding to the human immunodeficiency virus (HIV)-1 transactivator of transcription (Tat) protein, by modulating ROS homeostasis, mitogen-activated protein kinase (MAPK), NF- $\mathrm{B}$ and AP-1 signaling $(54,55)$. In summary, HDAC6 mediates HIV-1 Tat-induced pro-inflammatory response by regulating the MAPK, NF- $\kappa \mathrm{B}$ and AP-1 signaling pathways in astrocytes. Studies showed that hindsiipropane B inhibits the expression of HDAC6 induced by HIV-1 Tat, and subsequently inhibiting the expression of CCL2, CXCL8 and
CXCL10 mediated by the former. Another study confirmed that hindsiipropane B inhibits the expression of CCL2, CXCL8 and CXCL10 by inhibiting the HDAC6-NADPH oxidase-ROS-MAPK-NF- $\kappa \mathrm{B}-\mathrm{AP}-1$ axis in astrocytes (56). Zhang et al $(50,57)$ similarly found that selective HDAC6 inhibition decreases TNF- $\alpha$, IL- $1 \beta$ and IL- 6 expression in both LPS-activated RAW264.7 cells and a murine model of acute liver failure by modulating oxidative stress and toll-like receptor 4(TLR4)-MAPK-NF- $\kappa \mathrm{B}$ signaling. Liu et al (58) and Wang et al (59) have since confirmed that HDAC6 inhibition protects against LPS-induced inflammation by suppressing $\mathrm{NF}-\kappa \mathrm{B}$ signaling. It also decreases IL-1 $\beta$ expression, as well as caspase-1 cleavage and activation.

Interferon regulatory factor 3 (IRF-3) is a transcription factor that critically mediates interferon (IFN) secretion during inflammation (60). Interestingly, Nusinzon and Horvath (61) showed that HDAC6 modulates IRF-3 activity by deacetylating its coactivator, $\beta$-catenin. Moreover, Chattopadhyay et al (62) demonstrated that IRF-3, $\beta$-catenin and CBP form a stable complex in response to viral or bacterial infection-stimulated TLR-3 signaling to induce IFN production. Importantly, HDAC6 is required for the formation of this complex, since its inhibition modulates $\beta$-catenin acetylation, thereby suppressing the interaction of IRF-3 with CBP.

\section{HDAC6 inhibition restores endothelial barrier hyper-permeability}

The endothelial barrier is a semi-permeable membrane that maintains the intra- and extravascular balance of water and proteins (63). Its composition has previously been reported in detail; in brief, it comprises the cytoskeleton and adherents such as adherens junction (AJ) and tight junction (TJ) proteins, including claudins and occludins, as well as zonula occludens (ZO) (52) and local gap junction proteins (64). Endothelial barrier dysfunction plays an important role in the pathogenesis of sepsis-induced organ dysfunction; thus, endothelial barrier protection is a proposed therapeutic modality to treat sepsis. Nam et al (65) previously demonstrated that Clostridium difficile toxin A induces microtubule instability in a murine model of $C$. Difficile infection by activating HDAC6, which in turn modulates $\alpha$-tubulin deacetylation, thereby inducing acute inflammation. Moreover, it is now well known that HDAC6 inhibition promotes endothelial barrier hyper-permeability $(37-39,66,67)$.

HDAC6 modulates HSP90-dependent Rho-associated kinase (Rho-ROCK) activity. Hsp90 protein is a member of the heat shock molecular chaperone family that regulates protein conformation and activity, and regulates a variety of cell signaling pathways by controlling the abundance and activity of several important protein kinases and cell cycle-associated proteins, such as Rho GTPases and actin, which is a tripartite framework for effective vesicular transport. Rho GTPase is a subfamily of small GTP binding proteins in the Ras super family, composed of Cdc42, Rac1 and RhoA, which regulates actin dynamics in endothelial cells. For example, RhoA induces endothelial hyper-permeability by modulating actin stress fiber formation and cell contraction (68). ROCK increases actin filament crosslinking activity of myosin II 
through the phosphorylation-induced activation of MLC and inactivation of MLC phosphatase. Inhibition of Rho kinase, the downstream effector of RhoA, can protect HLMVECS from LPS-mediated high permeability and eliminate LPS-induced phosphorylation of MLC. Hsp90 plays an important and special role in regulating Rho activity and Rho-dependent actin cytoskeleton remodeling. Hsp90 can indirectly activate Rho GTPase by protecting functional activators from proteasome degradation (69). Consistent with these findings, the phosphorylation of MLC induced by RhoA is also inhibited by $17-\mathrm{AAG}$, a Hsp90 inhibitor. Studies have demonstrated that inhibition of Hsp90 can prevent and repair LPS-induced pulmonary endothelial barrier dysfunction by inhibiting RhoA activity and signal transduction (70). It is important to note that HSP90 is a substrate of HDAC6. A previous study by Joshi et al (37) proposed that HDAC6 inhibition protects against pulmonary endothelial barrier dysfunction and ALI by modulating HSP90; HDAC6 inhibition facilitates the acetylation of HSP90 at Lys294, which in turn suppresses HSP90-dependent Rho-ROCK activity and blocks MLC phosphorylation to provide protection against LPS-induced endothelial barrier dysfunction. However, more studies are required to verify this mechanism.

HDAC6 modulates the acetylation of $\alpha$-tubulin and $\beta$-catenin . Vascular endothelial (VE)-cadherin and $\beta$-catenin are major AJ components. The majority of $\beta$-catenin molecules localize to the cytoplasmic side of the membrane, where they contact VE-cadherin (71). In parallel, $\beta$-catenin degradation is controlled by its $\mathrm{N}$-terminal phosphorylation and ubiquitination; for example, ubiquitination at Lys49 triggers proteasomal $\beta$-catenin degradation (72). VE-cadherin contains five extracellular cadherin repeats, a transmembrane region, and a highly conserved cytoplasmic tail that has been shown to form a complex with $\beta$-catenin to maintain endothelial barrier integrity (73-75).

Microtubules and microfilaments are interacting cytoskeletal components (76,77). Microtubules are comprised of two globular proteins, $\alpha$ - and $\beta$-tubulin, which polymerize to regulate endothelial barrier function. Decreasing $\alpha$-tubulin acetylation has been shown to affect microtubule assembly, cytoskeletal stability and cell mobility (78). Conversely, tubulin acetylation prolongs the microtubule half-life, increases cytoskeletal stability, and renders the cytoskeleton more resistant to drug-induced depolymerization and disassembly (79). Previous studies have suggested that microtubule disassembly stimulates actin formation and increases MLC phosphorylation; in contrast, microtubule depolymerization likely blocks these effects $(80,81)$.

HDAC6 inhibition has been demonstrated to decrease sepsis-induced endothelial barrier hyper-permeability by restoring normal $\alpha$-tubulin and $\beta$-catenin acetylation patterns $(39,44)$. Specifically, HDAC6 inhibition allows $\beta$-catenin to be acetylated at Lys 49 , preventing its ubiquitination and increasing its accumulation at the cell membrane, thus promoting the formation of the VE-cadherin- $\beta$-catenin complex (82). Furthermore, HDAC6 inhibition has been suggested to modulate $\alpha$-tubulin and $\beta$-catenin acetylation by decreasing caspase-3 cleavage $(39,44)$. Decreased caspase-3 activation protects endothelial hyper-permeability, as evidenced by the fact that caspase- 3 inhibition restores endothelial barrier permeability in the lung tissue of septic mice (83). Increased caspase-3 activity likely contributes to endothelial barrier dysfunction by inducing endothelial cell apoptosis; however, it has also been reported to trigger the rearrangement of connexins, including VE-cadherin, ZO-1and claudin-5 (84-86).

\section{A promising and highly selective HDAC6 inhibitor, TubA}

In recent years, with an increase in the understanding of HDAC6 and its roles in different diseases, a large number of experimental studies on HDAC6 inhibitors have been reported. There is an increasing number of studies on the role of HDAC6 inhibition in inflammation, one of which involves the following: Computer-aided identification of new selective inhibitors of HDAC6 with anti-sepsis activity, development of new HDAC inhibitors after virtual screening based on chemical databases, compound 9A [(E)-n-hydroxy-4-(2-styrylthiazol-4-yl] was identified as a HDAC6 selective inhibitor $\left(\mathrm{IC}_{50}\right.$ value of HDAC6 is $0.199 \mu \mathrm{m}$; HDAC, $8 \mu \mathrm{m}$ ). Compound 9A significantly increased the survival of mice with sepsis induced by LPS and inhibited the increase of TNF- $\alpha$ and IL- 6 mRNA expression induced by LPS (46).

CAY is also a highly selective HDAC6 inhibitor, which blocks the activation of NF- $\kappa$ B by inhibiting $\mathrm{I} \kappa \mathrm{B}$ phosphorylation in LPS-induced ALI, can decrease the activity of inflammatory corpuscles induced by LPS and decrease the cleavage and activation of IL-1 $\beta$ and caspase-1 (58).

TubA is considered to be a potent and highly selective HDAC6 inhibitor. Its $\mathrm{IC}_{50}$ value is $15 \mathrm{~nm}, 1,000$ times lower than that of other subtypes, except HDAC8 (57 times lower). TubA can improve the survival of mice with septicemic induced by lethal CLP (42). In a hemorrhagic shock model combined with CLP, the survival time of mice in HDAC6 group was also prolonged (45). The mechanism included changing the composition of circulating blood cells in lethal septicemia model (48). Compared with the CLP Group, the TubA treatment group recovered B lymphocytes and significantly increased the percentage of innate immune cells and macrophages. In addition, TubA could significantly decrease the bacterial load in the spleen and increase the phagocytic capacity of macrophages in RAW264.7 cells. In this lethal sepsis model, TubA significantly decreased the stress response and thymus and bone marrow atrophy (51). The apoptosis of spleen cells also decreased. WT mice treated with TubA were partially protected from vascular leakage and inflammation caused by HKSA or methicillin-resistant Staphylococcus aureus (MRSA) (40). Thus, TubA plays an important function in endothelial cell barrier protection, and the mechanisms include an increase in the acetylation of $\alpha$-tubulin and $\beta$-catenin and a decrease in the activation of caspase- 3 as previously mentioned.

A brief introduction to the role of TubA in ARDS. As mentioned above, most of the experiments on HDAC6 inhibitors in vitro and in vivo have been conducted with TubA. Currently, TubA has been reported to prolong the survival period of septic mice, decrease the ALI from sepsis, and lower the bacterial load in spleen. It brings about these effects mainly by decreasing 
the inflammatory response, restoring endothelial cell barrier dysfunction, and decreasing the oxidative stress response, as well as pulmonary fibrosis, by inhibiting the apoptosis of pulmonary vascular endothelial cells (87).

Putative side effects of TubA. As the number of TubA studies increases, increasingly more attention is being given to its possible side effects. In one study, a relatively high dose was used (100 mg/kg body weight of mice) and no significant adverse effects were observed (88). However, in another study it was found that TubA exposure significantly decreased the formation of blastocysts in early embryos of mice, and confocal microscopy showed that chromosome aggregation failed to occur in mice embryos treated with HDAC6 inhibitors. In addition, the inhibition of HDAC6 induced an excessive production of ROS. An increase in the accumulation of phosphorylated $\gamma$-H2AX was also observed in the embryos after TubA intervention indicating an increase in DNA damage and blastocyst cell apoptosis (89). TubA interfered with and halted mouse oocyte meiosis by regulating several key histones (H4K16 acetylation and h3t3 and H3S10 phosphorylation) and messenger RNA (ccnb1, CDK2, Smad3 and YWHAZ methylation-associated genes DNMT1 and DNMT3b), and by interfering with the organization of spindles of chromosomes, as well as the attachment of mitotic microtubules. The first polar body of mouse blastocyst oocyte could not be expelled after TubA treatment. However, it has been proved that homozygous knockout of HDAC6 (KO) mice are viable and fertile. Further studies have confirmed that the HDAC6 protein and mRNA levels in mouse oocytes treated with TubA were significantly lower. The SIRT2, HDAC6, SIRT6 and SIRT7 mRNA levels were also significantly decreased. The mRNA expression levels of Cdk1, Cdk2, Cdk4, Cdk6, Cdc25B, and other cell cycle-associated kinases decreased significantly. These abnormalities were associated with meiosis and cell aging. The abnormal meiotic maturation and cell senescence induced by TubA may be the result of its interaction with HDAC and sirtuin (90). It is presumed that TubA can be used in the treatment of ARDS induced by sepsis, but effects of HDAC6 on the reproductive function of female should be of caution.

\section{Conclusion}

Inflammation and endothelial barrier dysfunction are important processes that contribute to the pathogenesis of sepsis-induced ARDS. HDAC6 has been reported to mediate inflammation, as well as both the innate and adaptive immunity, including inflammatory cell recruitment and bacterial clearance. Accordingly, HDAC6 inhibition using an inhibitor such as TubA increases the survival of septic mice by decreasing the LPS-induced macrophage and epithelial cell inflammation. It is proposed that HDAC6 inhibition predominantly ameliorates sepsis-induced ARDS by modulating the innate and adaptive immunity and the transcription of pro-inflammatory genes to directly attenuate inflammation. HDAC6 inhibition also protects endothelial barrier function via its effects on its target substrates by inducing $\alpha$-tubulin and $\beta$-catenin acetylation and increasing membrane localization of $\beta$-catenin, thereby leading to the stabilization of microtubule and AJs. It also prevents caspase-3 activation, maintains lung endothelial cell-cell junctions, and inhibits actin stress fiber formation and decreases MLC phosphorylation via modulating HSP90. These findings support that HDAC6 inhibition is a promising potential therapeutic target to treat sepsis-induced ARDS. However, the negative effects, if any, of HDAC6 inhibitors on embryo sac and oocytes need to be taken into account.

\section{Acknowledgements}

Not applicable.

\section{Funding}

This work was supported by the National Natural Science Foundation of China (grant no. 81800080).

\section{Availability of data and materials}

All data generated or analyzed during this study are included in this published article.

\section{Authors' contributions}

JYu analyzed and interpreted the data and final approval of the version to be published. JYa analyzed and interpreted the data and was a major contributor in writing the manuscript. QZ was a contributor in writing the manuscript. YW and DQ contributed to literature review. All authors read and approved the final manuscript.

\section{Ethics approval and consent to participate}

Not applicable.

\section{Patient consent for publication}

Not applicable.

\section{Competing interests}

The authors declare that they have no competing interests.

\section{References}

1. Ashbaugh DG, Bigelow DB, Petty TL and Levine BE: Acute respiratory distress in adults. Lancet 2: 319-323, 1967.

2. Ferguson ND, Fan E, Camporota L, Antonelli M, Anzueto A, Beale R, Brochard L, Brower R, Esteban A, Gattinoni L, et al: The Berlin definition of ARDS: An expanded rationale, justification, and supplementary material. Intensive Care Med 38: 1573-1582, 2012.

3. Bellani G, Laffey JG, Pham T, Fan E, Brochard L, Esteban A, Gattinoni L, van Haren F, Larsson A, McAuley DF, et al; LUNG SAFE Investigators; ESICM Trials Group: Epidemiology, patterns of care, and mortality for patients with acute respiratory distress syndrome in intensive care units in 50 countries. JAMA 315: 788-800, 2016.

4. Herridge MS, Tansey CM, Matté A, Tomlinson G, Diaz-Granados N, Cooper A, Guest CB, Mazer CD, Mehta S, Stewart TE, et al; Canadian Critical Care Trials Group: Functional disability 5 years after acute respiratory distress syndrome. N Engl J Med 364: 1293-1304, 2011.

5. Han S and Mallampalli RK: The acute respiratory distress syndrome: From mechanism to translation. J Immunol 194: 855-860, 2015. 
6. Griffiths MJD, McAuley DF, Perkins GD, Barrett N, Blackwood B, Boyle A, Chee N, Connolly B, Dark P, Finney S, et al: Guidelines on the management of acute respiratory distress syndrome. BMJ Open Respir Res 6: e000420, 2019.

7. Walkey AJ, Del Sorbo L, Hodgson CL, Adhikari NKJ, Wunsch H, Meade MO, Uleryk E, Hess D, Talmor DS, Thompson BT, et al: Higher PEEP versus Lower PEEP Strategies for Patients with Acute Respiratory Distress Syndrome. A Systematic Review and Meta-Analysis. Ann Am Thorac Soc 14 (Suppl 4): S297-S303, 2017.

8. Hodgson CL, Cooper DJ, Arabi Y, King V, Bersten A, Bihari S, Brickell K, Davies A, Fahey C, Fraser J, et al: Maxima Recruitment Open Lung Ventilation in Acute Respiratory Distress Syndrome (PHARLAP). A Phase II, Multicenter Randomized Controlled Clinical Trial. Am J Respir Crit Care Med 200: 1363-1372, 2019.

9. Steinberg KP, Hudson LD, Goodman RB, Hough CL, Lanken PN, Hyzy R, Thompson BT and Ancukiewicz M; National Heart, Lung, and Blood Institute Acute Respiratory Distress Syndrome (ARDS) Clinical Trials Network: Efficacy and safety of corticosteroids for persistent acute respiratory distress syndrome. $\mathrm{N}$ Engl J Med 354: 1671-1684, 2006.

10. Peck TJ and Hibbert KA: Recent advances in the understanding and management of ARDS. F1000 Res 8: 8, 2019.

11. Villar J, Ferrando C, Martínez D, Ambrós A, Muñoz T, Soler JA, Aguilar G, Alba F, González-Higueras E, Conesa LA, et al; dexamethasone in ARDS network: Dexamethasone treatment for the acute respiratory distress syndrome: A multicentre, randomised controlled trial. Lancet Respir Med 8: 267-276, 2020.

12. Rubenfeld GD, Caldwell E, Peabody E, Weaver J, Martin DP, Neff M, Stern EJ and Hudson LD: Incidence and outcomes of acute lung injury. N Engl J Med 353: 1685-1693, 2005.

13. Ito K, Caramori G, Lim S, Oates T, Chung KF, Barnes PJ and Adcock IM: Expression and activity of histone deacetylases in human asthmatic airways. Am J Respir Crit Care Med 166 392-396, 2002.

14. Sadoul K, Boyault C, Pabion M and Khochbin S: Regulation of protein turnover by acetyltransferases and deacetylases. Biochimie 90: 306-312, 2008.

15. Dekker FJ and Haisma HJ: Histone acetyl transferases as emerging drug targets. Drug Discov Today 14: 942-948, 2009.

16. Zou H, Wu Y, Navre M and Sang BC: Characterization of the two cataly tic domains in histone deacetylase 6. Biochem Biophys Res Commun 341: 45-50, 2006.

17. Zhang Y, Gilquin B, Khochbin S and Matthias P: Two catalytic domains are required for protein deacetylation. J Biol Chem 281: 2401-2404, 2006.

18. Schäfer S, Saunders L, Eliseeva E, Velena A, Jung M, Schwienhorst A, Strasser A, Dickmanns A, Ficner R and Schlimme S: Phenylalanine-containing hydroxamic acids as selective inhibitors of class IIb histone deacetylases (HDACs). Bioorg Med Chem 16: 2011-2033, 2008.

19. Zhang X, Yuan Z, Zhang Y, Yong S, Salas-Burgos A, Koomen J, Olashaw N, Parsons JT, Yang XJ, Dent SR, et al: HDAC6 modulates cell motility by altering the acetylation level of cortactin. Mol Cell 27: 197-213, 2007.

20. Bali P, Pranpat M, Bradner J, Balasis M, Fiskus W, Guo F, Rocha K, Kumaraswamy S, Boyapalle S, Atadja P, et al: Inhibition of histone deacetylase 6 acetylates and disrupts the chaperone function of heat shock protein 90: A novel basis for antileukemia activity of histone deacetylase inhibitors. J Biol Chem 280: 26729-26734, 2005.

21. Parab S, Shetty O, Gaonkar R, Balasinor N, Khole V and Parte P: Correction to: HDAC6 deacetylates alpha tubulin in sperm and modulates sperm motility in Holtzman rat. Cell Tissue Res 371 375,2018

22. Wang XX, Wan RZ and Liu ZP: Recent advances in the discovery of potent and selective HDAC6 inhibitors. Eur J Med Chem 143: 1406-1418, 2018

23. Jia YJ, Liu ZB, Wang WG, Sun CB, Wei P, Yang YL, You MJ, $\mathrm{Yu}$ BH, Li XQ and Zhou XY: HDAC6 regulates microRNA-27b that suppresses proliferation, promotes apoptosis and target MET in diffuse large B-cell lymphoma. Leukemia 32: 703-711, 2018.

24. Vogl DT, Raje N, Jagannath S, Richardson P, Hari P, Orlowski R, Supko JG, Tamang D, Yang M, Jones SS, et al: Ricolinostat, the first selective histone deacetylase 6 inhibitor, in combination with bortezomib and dexamethasone for relapsed or refractory multiple myeloma. Clin Cancer Res 23: 3307-3315, 2017.
25. Yee AJ, Bensinger WI, Supko JG, Voorhees PM, Berdeja JG, Richardson PG, Libby EN, Wallace EE, Birrer NE, Burke JN, et al: Ricolinostat plus lenalidomide, and dexamethasone in relapsed or refractory multiple myeloma: A multicentre phase $1 \mathrm{~b}$ trial. Lancet Oncol 17: 1569-1578, 2016.

26. Tu Y, Hershman DL, Bhalla K, Fiskus W, Pellegrino CM, Andreopoulou E, Makower D, Kalinsky K, Fehn K, Fineberg S, et al: A phase I-II study of the histone deacetylase inhibitor vorinostat plus sequential weekly paclitaxel and doxorubicin-cyclophosphamide in locally advanced breast cancer. Breast Cancer Res Treat 146: 145-152, 2014.

27. Halili MA, Andrews MR, Labzin LI, Schroder K, Matthias G, Cao C, Lovelace E, Reid RC, Le GT, Hume DA, et al: Differential effects of selective HDAC inhibitors on macrophage inflammatory responses to the Toll-like receptor 4 agonist LPS. J Leukoc Biol 87: 1103-1114, 2010.

28. Estenssoro E and Dubin A: Acute respiratory distress syndrome. Medicina (B Aires) 76: 235-241, 2016 (In Spanish).

29. Kasznica J, Helmann M, Collins JP and Akhtar R: Bilateral Ebstein-like anomaly with atrial septal defect. Jpn Heart J 36: 119-125, 1995.

30. Fu P, Murley JS, Grdina DJ, Birukova AA and Birukov KG: Induction of cellular antioxidant defense by amifostine improves ventilator-induced lung injury. Crit Care Med 39: 2711-2721, 2011.

31. Kratzer E, Tian Y, Sarich N, Wu T, Meliton A, Leff A and Birukova AA: Oxidative stress contributes to lung injury and barrier dysfunction via microtubule destabilization. Am J Respir Cell Mol Biol 47: 688-697, 2012.

32. Zhao B, Gao W, Gao X, Leng Y, Liu M, Hou J and Wu Y: Sulforaphane attenuates acute lung injury by inhibiting oxidative stress via Nrf2/HO-1 pathway in a rat sepsis model. Int J Clin Exp Pathol 10: 9021-9028, 2017.

33. Peng X, Hassoun PM, Sammani S, McVerry BJ, Burne MJ, Rabb H, Pearse D, Tuder RM and Garcia JG: Protective effects of sphingosine 1-phosphate in murine endotoxin-induced inflammatory lung injury. Am J Respir Crit Care Med 169: 1245-1251, 2004.

34. Kong J, Zhu X, Shi Y, Liu T, Chen Y, Bhan I, Zhao Q, Thadhani $\mathrm{R}$ and Li YC: VDR attenuates acute lung injury by blocking Ang-2-Tie-2 pathway and renin-angiotensin system. Mol Endocrinol 27: 2116-2125, 2013.

35. Thangavel J, Malik AB, Elias HK, Rajasingh S, Simpson AD, Sundivakkam PK, Vogel SM, Xuan YT, Dawn B and Rajasingh J: Combinatorial therapy with acetylation and methylation modifiers attenuates lung vascular hyperpermeability in endotoxemia-induced mouse inflammatory lung injury. Am J Pathol 184: 2237-2249, 2014.

36. Xu Q, Liu J, Wang Z, Guo X, Zhou G, Liu Y, Huang Q and Su L: Heat stress-induced disruption of endothelial barrier function is via PAR1 signaling and suppressed by Xuebijing injection. PLoS One 10: e0118057, 2015.

37. Joshi AD, Barabutis N, Birmpas C, Dimitropoulou C, Thangjam G, Cherian-Shaw M, Dennison J and Catravas JD: Histone deacetylase inhibitors prevent pulmonary endothelial hyperpermeability and acute lung injury by regulating heat shock protein 90 function. Am J Physiol Lung Cell Mol Physiol 309: L1410-L1419, 2015.

38. Borgas D, Chambers E, Newton J, Ko J, Rivera S, Rounds S and Lu Q: Cigarette Smoke Disrupted Lung Endothelial Barrier Integrity and Increased Susceptibility to Acute Lung Injury via Histone Deacetylase 6. Am J Respir Cell Mol Biol 54: 683-696, 2016.

39. Yu J, Ma Z, Shetty S, Ma M and Fu J: Selective HDAC6 inhibition prevents TNF- $\alpha$-induced lung endothelial cell barrier disruption and endotoxin-induced pulmonary edema. Am J Physiol Lung Cell Mol Physiol 311: L39-L47, 2016.

40. Karki P, Ke Y, Tian Y, Ohmura T, Sitikov A, Sarich N, Montgomery CP and Birukova AA: Staphylococcus aureus-induced endothelial permeability and inflammation are mediated by microtubule destabilization. J Biol Chem 294: 3369-3384, 2019.

41. Rosenjack J, Hodges CA, Darrah RJ and Kelley TJ: HDAC6 depletion improves cystic fibrosis mouse airway responses to bacterial challenge. Sci Rep 9: 10282, 2019.

42. Deng Q, Zhao T, Pan B, Dennahy IS, Duan X, Williams AM, Liu B, Lin N, Bhatti UF, Chen E, et al: Protective Effect of Tubastatin A in CLP-Induced Lethal Sepsis. Inflammation 41: 2101-2109, 2018. 
43. Li Y, Zhao T, Liu B, Halaweish I, Mazitschek R, Duan X and Alam HB: Inhibition of histone deacetylase 6 improves long-term survival in a lethal septic model. J Trauma Acute Care Surg 78 378-385, 2015

44. Yu J, Ma M, Ma Z and Fu J: HDAC6 inhibition prevents TNF- $\alpha$-induced caspase 3 activation in lung endothelial cell and maintains cell-cell junctions. Oncotarget 7: 54714-54722, 2016.

45. Cheng X, Liu Z, Liu B, Zhao T, Li Y and Alam HB: Selective histone deacetylase 6 inhibition prolongs survival in a lethal two-hit model. J Surg Res 197: 39-44, 2015.

46. Yoo J, Kim SJ, Son D, Seo H, Baek SY, Maeng CY, Lee C, Kim IS, Jung YH, Lee SM, et al: Computer-aided identification of new histone deacetylase 6 selective inhibitor with anti-sepsis activity. Eur J Med Chem 116: 126-135, 2016.

47. Moreno-Gonzalo O, Mayor F Jr and Sánchez-Madrid F: HDAC6 at Crossroads of Infection and Innate Immunity. Trends Immunol 39: 591-595, 2018.

48. Zhao T, Li Y, Liu B, Pan B, Cheng X, Georgoff P and Alam HB: Inhibition of histone deacetylase 6 restores innate immune cells in the bone marrow in a lethal septic model. J Trauma Acute Care Surg 80: 34-40, discussion 40-41, 2016

49. Yan B, Xie S, Liu Y, Liu W, Li D, Liu M, Luo HR and Zhou J: Histone deacetylase 6 modulates macrophage infiltration during inflammation. Theranostics 8: 2927-2938, 2018.

50. Zhang WB, Yang F, Wang Y, Jiao FZ, Zhang HY, Wang LW and Gong ZJ: Inhibition of HDAC6 attenuates LPS-induced inflammation in macrophages by regulating oxidative stress and suppressing the TLR4-MAPK/NF- $\mathrm{B}$ pathways. Biomed Pharmacother 117: 109166, 2019.

51. Zhao T, Li Y, Bronson RT, Liu B, Velmahos GC and Alam HB: Selective histone deacetylase-6 inhibition attenuates stress responses and prevents immune organ atrophy in a lethal septic model. Surgery 156: 235-242, 2014.

52. González-Mariscal L, Tapia R and Chamorro D: Crosstalk of tight junction components with signaling pathways. Biochim Biophys Acta 1778: 729-756, 2008.

53. Di Liddo R, Valente S, Taurone S, Zwergel C, Marrocco B, Turchetta R, Conconi MT, Scarpa C, Bertalot T, Schrenk S, et al: Histone deacetylase inhibitors restore IL-10 expression in lipopolysaccharide-induced cell inflammation and reduce IL-1 $\beta$ and IL-6 production in breast silicone implant in C57BL/6J wild-type murine model. Autoimmunity: Jan 20, 2016 (Epub ahead of print).

54. Youn GS, Lee KW, Choi SY and Park J: Overexpression of HDAC6 induces pro-inflammatory responses by regulating ROS-MAPK-NF- $\mathrm{KB} / \mathrm{AP}-1$ signaling pathways in macrophages. Free Radic Biol Med 97: 14-23, 2016.

55. Youn GS, Ju SM, Choi SY and Park J: HDAC6 mediates HIV-1 tat-induced proinflammatory responses by regulating MAPK-NF-kappaB/AP-1 pathways in astrocytes. Glia 63 . 1953-1965, 2015

56. Jo H, Jang HY, Youn GS, Kim D, Lee CY, Jang JH, Choi SY, Jun JG and Park J: Hindsiipropane B alleviates HIV-1 Tat-induced inflammatory responses by suppressing HDAC6-NADPH oxidase-ROS axis in astrocytes. BMB Rep 51: 394-399, 2018

57. Zhang WB, Zhang HY, Jiao FZ, Wang LW, Zhang $\mathrm{H}$ and Gong ZJ: Histone deacetylase 6 inhibitor ACY-1215 protects against experimental acute liver failure by regulating the TLR4-MAPK/NF- $\mathrm{B}$ pathway. Biomed Pharmacother 97: 818-824, 2018

58. Liu L, Zhou X, Shetty S, Hou G, Wang Q and Fu J: HDAC6 inhibition blocks inflammatory signaling and caspase-1 activation in LPS-induced acute lung injury. Toxicol Appl Pharmacol 370: 178-183, 2019.

59. Wang J, Zhao L, Wei Z, Zhang X, Wang Y, Li F, Fu Y and Liu B: Inhibition of histone deacetylase reduces lipopolysaccharide-induced-inflammation in primary mammary epithelial cells by regulating ROS-NF-KB signaling pathways Int Immunopharmacol 56: 230-234, 2018.

60. Hiscott J: Convergence of the NF-kappaB and IRF pathways in the regulation of the innate antiviral response. Cytokine Growth Factor Rev 18: 483-490, 2007.

61. Nusinzon I and Horvath CM: Positive and negative regulation of the innate antiviral response and beta interferon gene expression by deacetylation. Mol Cell Biol 26: 3106-3113, 2006.

62. Chattopadhyay S, Fensterl V, Zhang Y, Veleeparambil M Wetzel JL and Sen GC: Inhibition of viral pathogenesis and promotion of the septic shock response to bacterial infection by IRF-3 are regulated by the acetylation and phosphorylation of its coactivators. MBio 4: 4, 2013
63. Trani M and Dejana E: New insights in the control of vascular permeability: Vascular endothelial-cadherin and other players. Curr Opin Hematol 22: 267-272, 2015.

64. Rodrigues SF and Granger DN: Blood cells and endothelial barrier function. Tissue Barriers 3: e978720, 2015.

65. Nam HJ, Kang JK, Kim SK, Ahn KJ, Seok H, Park SJ, Chang JS, Pothoulakis C, Lamont JT and Kim H: Clostridium difficile toxin A decreases acetylation of tubulin, leading to microtubule depolymerization through activation of histone deacetylase 6 , and this mediates acute inflammation. J Biol Chem 285: 32888-32896, 2010.

66. Saito S, Lasky JA, Guo W, Nguyen H, Mai A, Danchuk S, Sullivan DE and Shan B: Pharmacological inhibition of HDAC6 attenuates endothelial barrier dysfunction induced by thrombin. Biochem Biophys Res Commun 408: 630-634, 2011.

67. Wang F, Zheng L, Yi Y, Yang Z, Qiu Q, Wang X, Yan W, Bai P, Yang J, Li D, et al: SKLB-23bb, A HDAC6-Selective Inhibitor, Exhibits Superior and Broad-Spectrum Antitumor Activity via Additionally Targeting Microtubules. Mol Cancer Ther 17: 763-775, 2018

68. Majolée J, Pronk MCA, Jim KK, van Bezu JSM, van der Sar AM, Hordijk PL and Kovačević I: CSN5 inhibition triggers inflammatory signaling and Rho/ROCK-dependent loss of endothelial integrity. Sci Rep 9: 8131, 2019.

69. Croisé P, Estay-Ahumada C, Gasman S and Ory S: Rho GTPases, phosphoinositides, and actin: A tripartite framework for efficient vesicular trafficking. Small GTPases 5: e29469, 2014.

70. Joshi AD, Dimitropoulou C, Thangjam G, Snead C, Feldman S, Barabutis N, Fulton D, Hou Y, Kumar S, Patel V, et al: Heat shock protein 90 inhibitors prevent LPS-induced endothelial barrier dysfunction by disrupting RhoA signaling. Am J Respir Cell Mol Biol 50: 170-179, 2014.

71. Valenta T, Hausmann G and Basler K: The many faces and functions of $\beta$-catenin. EMBO J 31: 2714-2736, 2012.

72. Winer IS, Bommer GT, Gonik N and Fearon ER: Lysine residues Lys-19 and Lys-49 of beta-catenin regulate its levels and function in $\mathrm{T}$ cell factor transcriptional activation and neoplastic transformation. J Biol Chem 281: 26181-26187, 2006.

73. Sauteur L, Krudewig A, Herwig L, Ehrenfeuchter N, Lenard A, Affolter $\mathrm{M}$ and Belting HG: Cdh5/VE-cadherin promotes endothelial cell interface elongation via cortical actin polymerization during angiogenic sprouting. Cell Rep 9: 504-513, 2014.

74. Dejana E and Vestweber D: The role of VE-cadherin in vascular morphogenesis and permeability control. Prog Mol Biol Transl Sci 116: 119-144, 2013

75. Huber AH and Weis WI: The structure of the beta-catenin/ E-cadherin complex and the molecular basis of diverse ligand recognition by beta-catenin. Cell 105: 391-402, 2001.

76. D'Alessandro M, Hnia K, Gache V, Koch C, Gavriilidis C, Rodriguez D, Nicot AS, Romero NB, Schwab Y, Gomes E, et al: Amphiphysin 2 orchestrates nucleus positioning and shape by linking the nuclear envelope to the actin and microtubule cytoskeleton. Dev Cell 35: 186-198, 2015.

77. Coles $\mathrm{CH}$ and Bradke F: Coordinating neuronal actin-microtubule dynamics. Curr Biol 25: R677-R691, 2015.

78. Hubbert C, Guardiola A, Shao R, Kawaguchi Y, Ito A, Nixon A, Yoshida M, Wang XF and Yao TP: HDAC6 is a microtubule-associated deacetylase. Nature 417: 455-458, 2002.

79. Matsuyama A, Shimazu T, Sumida Y, Saito A, Yoshimatsu Y, Seigneurin-Berny D, Osada H, Komatsu Y, Nishino N, Khochbin S, et al: In vivo destabilization of dynamic microtubules by HDAC6-mediated deacetylation. EMBO J 21: 6820-6831, 2002

80. Shivanna M and Srinivas SP: Microtubule stabilization opposes the (TNF-alpha)-induced loss in the barrier integrity of corneal endothelium. Exp Eye Res 89: 950-959, 2009.

81. Bogatcheva NV and Verin AD: The role of cytoskeleton in the regulation of vascular endothelial barrier function. Microvasc Res 76: 202-207, 2008.

82. Iaconelli J, Huang JH, Berkovitch SS, Chattopadhyay S, Mazitschek R, Schreiber SL, Haggarty SJ and Karmacharya R: HDAC6 inhibitors modulate Lys49 acetylation and membrane localization of $\beta$-catenin in human iPSC-derived neuronal cells. ACS Chem Biol 10: 883-890, 2015.

83. Matsuda N, Takano Y,Kageyama S, Hatakeyama N, Shakunaga K, Kitajima I, Yamazaki M and Hattori Y: Silencing of caspase- 8 and caspase- 3 by RNA interference prevents vascular endothelial cell injury in mice with endotoxic shock. Cardiovasc Res 76: 132-140, 2007. 
84. Sawant DA, Tharakan B, Tobin RP, Reilly J, Hunter FA, Newell MK Smythe WR and Childs EW: Microvascular endothelial cell hyperpermeability induced by endogenous caspase 3 activator staurosporine. J Trauma Acute Care Surg 74: 516-523, 2013.

85. Lopez-Ramirez MA, Fischer R, Torres-Badillo CC, Davies HA, Logan K, Pfizenmaier K, Male DK, Sharrack B and Romero IA: Role of caspases in cytokine-induced barrier breakdown in human brain endothelial cells. J Immunol 189: 3130-3139, 2012.

86. Zehendner CM, Librizzi L, de Curtis M, Kuhlmann CR and Luhmann HJ: Caspase-3 contributes to ZO-1 and Cl-5 tight-junction disruption in rapid anoxic neurovascular unit damage. PLoS One 6: e16760, 2011.

87. Leyk J, Daly C, Janssen-Bienhold U, Kennedy BN and Richter-Landsberg C: HDAC6 inhibition by tubastatin A is protective against oxidative stress in a photoreceptor cell line and restores visual function in a zebrafish model of inherited blindness. Cell Death Dis 8: e3028, 2017.
88. Wang X, Tang X, Zhou Z and Huang Q: Histone deacetylase 6 inhibitor enhances resistance to Mycobacterium tuberculosis infection through innate and adaptive immunity in mice. Pathog Dis 76: 76, 2018.

89. Wang H, Ling L, Ai L and Bai L: HDAC6 inhibition induces the failure of mouse early embryonic development. J Cell Physiol 234: 8752-8759, 2019.

90. Choi YJ, Kang MH, Hong K and Kim JH: Tubastatin A inhibits HDAC and Sirtuin activity rather than being a HDAC6-specific inhibitor in mouse oocytes. Aging (Albany NY) 11: 1759-1777, 2019. 